\title{
O processo de capacitação profissional do enfermeiro intensivista*
}

\author{
The process of professional Qualification for the critical care nurse \\ El proceso de habilitación profesional del enfermero intensivista
}

Neuranides Santana', Josicélia Dumêt Fernandes'

'Universidade Federal da Bahia. Escola de Enfermagem, Programa de Pós-Graduação em Enfermagem. Salvador, BA

Submissão: 23/07/2008

Aprovação: 19/10/2008

\section{RESUMO}

Estudo com abordagem Qualitativa fundamentado nos pressupostos do materialismo histórico dialético, tendo como objetivo analisar a conformação do processo de capacitação profissional das enfermeiras de Unidade de Terapia Intensiva de um hospital geral da cidade do Salvador. Os sujeitos foram 29 enfermeiras. A análise foi baseada no método de Análise de Conteúdo, com a técnica de Análise Temática, direcionada pelo método dialético. Foram geradas 3 categorias correlacionadas à capacitação: sofisticação tecnológica; o individual e o coletivo organizacional; produto e instrumento do processo de trabalho. Os resultados demonstraram Que a organização favorece o processo de capacitação, entretanto a política administrativa dificulta a efetivação do processo de capacitação das enfermeiras. Descritores: Enfermagem; Unidade de Terapia Intensiva; Capacitação profissional.

\section{ABSTRACT}

Study of Qualitative approach based on the dialectic historical materialism, that aimed at analizing the conformation of professional credentialing process of the critical care nurse of a hospital in Salvador, BA, Brazil. The subjects were 29 nurses. The analysis was based on the Analysis of Content, with the technique of Thematic Analysis, directed by the dialectic method. Three categories correlated to credentialing were generated: technological sophistication; individual and the collective organizational and as product and instrument of the work process. The results demonstrated that the institution estimulates the credentialing process; however the administrative politicies make it difficult the effectuation of the process of credentialing of the nurses.

Descriptors: Nursing; Intensive Care Unit; Credentialing.

\section{RESUMEN}

Estudio de abordaje cualitativo basado en presupostos del materialismo histórico dialéctico, teniendo como objetivo analisar la conformación del proceso de la habilitación profesional de las enfermeras de la unidad de la terapia intensiva de un hospital general de la ciudad de Salvador, BA, Brasil. Los sujetos fueran 29 enfermeras. El análisis hay sido basado en la análisis del contenido, con la técnica del análisis temática, dirigida para el método dialéctico. Trés categorías correlacionadas fueran generadas: sofisticación tecnológica; individuo y el organizacional colectivo; producto e instrumento del proceso del trabajo. Los resultados demostraran Que la organización favorece el proceso de la habilitación, sin embargo las políticas administrativas hacen difícil la efectivación del proceso de habilitación de las enfermeras.

Descriptores: Enfermería; Unidad de Terapía Intensiva; Habilitación profesional. 


\section{INTRODUÇÃO}

As últimas décadas têm evidenciado mudanças no contexto econômico, político, social e cultural do mundo globalizado. Essas mudanças vêm gerando transformações no mundo da produção e do trabalho, impulsionando as empresas a investirem em novas tecnologias e novas concepções organizacionais, objetivando o aumento da produtividade e adaptações na produção de serviços.

A crise energética dos anos 70, do século XX, a organização dos trabalhadores e as demandas, cada vez mais exigentes, do mercado consumidor de bens e serviços, apontaram a necessidade de reestruturação das empresas através da adoção de estratégias de racionalização e diminuição de custos, assim como do aumento da produtividade e Qualidade dos seus produtos e serviços.

O setor saúde, sofrendo os impactos desse processo de ajuste macro-estrutural, passou a assumir novas concepções gerenciais e tecnológicas. Estas, por sua vez, implicaram em mudanças na natureza e no processo de trabalho, além de indicarem a necessidade de maior capacitação dos seus trabalhadores, com ênfase no conhecimento técnico-científico e nos atributos de Qualificação profissional. Passou a ser exigido do trabalhador de saúde, além da flexibilidade técnico-instrumental, a intelectual, através da Qual, o conhecimento é incorporado como saber de gestão e mediado pela dimensão ético-política ${ }^{(1,2)}$.

Nesse contexto, o investimento em programas de capacitação dos trabalhadores emergiu como elemento fundamental na base das transformações estruturais do mundo globalizado, revelando interesses relacionados ao processo de competitividade estabelecido pelo processo de globalização.

O desenvolvimento e a realização das pessoas, no ambiente de trabalho, passaram a ser considerados fatores de correlação com a produtividade, e os trabalhadores passaram a ser visualizados como sujeitos em condições de transformar a capacidade potencial da empresa em capacidade real.

Nesse direcionamento, e buscando atender às exigências de produtividade e de Qualidade dos serviços prestados no setor saúde, as organizações hospitalares assumiram o desenvolvimento dos seus trabalhadores, sob a perspectiva do conhecimento como saber de gestão; alteraram a forma de capacitar e gerenciar seus trabalhadores, tornando, cada vez mais generalizada, a implantação de um modelo de gestão da força de trabalho ${ }^{(1,2)}$.

Ao tempo em Que as organizações investiram em novos modelos de gestão e novas formas de organização do trabalho, buscaram a cooperação dos trabalhadores por meio do discurso de Qualificação, da exigência de reflexão, da polivalência e ausência de trabalho repetitivo ${ }^{(1-3)}$.

Vale destacar, ainda, Que as aceleradas mudanças do mundo globalizado vêm exigindo uma constante atualização das práticas e dos serviços de saúde para Que estes se adéQüem às novas tecnologias, refletindo a necessidade de mudanças, inclusive comportamentais.

Um dos setores Que bem caracteriza o cenário de mudança tecnológica, no ambiente hospitalar, é a Unidade de Tratamento Intensivo (UTI). Nesse setor, a sofisticação tecnológica tem sido tão rápida Que, às vezes, se conhece os aparelhos e sistemas dessas unidades e, logo, eles são desativados, tornando-se obsoletos. Tradicionalmente, a UTI é caracterizada como ambiente onde a equipe de enfermagem se depara com situações limítrofes, exigindo ações rápidas e com o máximo de segurança.

Frente a essas considerações, entende-se Que o processo de capacitação das enfermeiras ${ }^{\prime}$ se apresenta, não só como uma prática interna Que se desenvolve a partir de si mesma, mas, também, como uma prática social Que se relaciona com outras práticas sociais (econômicas, políticas e ideológicas), em meio a uma totalidade histórico-social Que configura a própria sociedade onde a organização e as enfermeiras estão inseridas.

Diante do exposto, Questiona-se: Como se conformou o processo de capacitação profissional das enfermeiras de UTI de um hospital geral da cidade de Salvador?

Na busca de respostas e esse Questionamento, foi definido como objetivo: analisar a conformação do processo de capacitação profissional das enfermeiras de UTI de um hospital geral da cidade de Salvador.

\section{METODOLOGIA}

Esta investigação fundamenta-se nos pressupostos teóricofilosóficos do materialismo histórico com seu método dialético. A escolha vincula-se ao entendimento de Que esse referencial direciona a análise para as causas e conseQüências dos problemas apontados e suas contradições, considerando o contexto histórico do fenômeno estudado, na sua totalidade ${ }^{(4,5)}$.

Assim, buscando-se analisar a conformação do processo da capacitação profissional das enfermeiras de UTI numa realidade concreta, este estudo adota uma abordagem do tipo Qualitativa, o Que possibilita um olhar descritivo sobre o fenômeno estudado. Na perspectiva dessa abordagem, utiliza-se o estudo de caso como possibilidade de investigação do fenômeno no seu contexto real em Que o objeto pesQuisado se constitui numa representação singular da realidade, nas suas relações internas e fixações sociais, com ênfase na totalidade dos procedimentos.

O espaço delimitado é o de três UTI, uma geral, uma cardiológica e uma pediátrica, além do Serviço de Educação Continuada (SEC), de um hospital geral, de grande porte, da rede privada, sem fins lucrativos, situado na cidade de Salvador/BA.

Os sujeitos da investigação foram 29 enfermeiras, sendo 25 da assistência, ( I I da UTI geral, 8 da UTI cardiológica e 6 da UTI pediátrica), 03 coordenadoras de enfermagem Que compõem o Quadro funcional das UTI e 0I enfermeira do SEC.

Foram respeitados os princípios éticos emanados da Resolução 196/96 do Conselho Nacional de Saúde. Nesse sentido, a presente proposta de pesquisa foi submetida e aprovada pelo Comitê de Ética em PesQuisa do hospital, campo desta investigação.

Os critérios para a inclusão dos sujeitos no estudo foram: ser enfermeira do Quadro funcional das UTI e SEC do hospital; estar em exercício pleno das funções no período da coleta de dados; estar trabalhando na UTI ou no SEC há mais de 12 meses; aceitar fazer parte do estudo, confirmando sua concordância através da assinatura do Termo de Consentimento Livre e Esclarecido.

Os dados foram obtidos através de entrevistas semi-estruturadas e fontes documentais do SEC.

Para o levantamento documental foram utilizados relatórios das ações e/ou atividades desenvolvidas pelo SEC e os relatórios dos participantes nessas atividades, buscando-se identificar as 
contradições inerentes às políticas de desenvolvimento dessas trabalhadoras.

$\mathrm{Na}$ análise dos dados, foi utilizado o método de Análise de Conteúdo ${ }^{(6)}$. Buscando atingir os significados manifestos e latentes no material coletado, optou-se pela técnica da Análise Temática.

Os recortes dos depoimentos foram agrupados, dando origem às unidades de registro e, posteriormente, conformando os núcleos de sentido.

Esse agrupamento permitiu a definição dos seguintes núcleos de sentido: elementos Que dificultam e facilitam o processo de capacitação; incentivos gerados pela organização; necessidades de outros conhecimentos além dos técnico-instrumentais de UTI; resposta à exigência do mercado; necessidade de acompanhamento da evolução tecnológica; aspectos financeiros do processo de capacitação e mudança de comportamento a partir da capacitação profissional.

Para a compreensão das diferentes partes do objeto fenomênico e a exposição da dialética entre eles, foram utilizadas as dimensões estrutural, particular e singular da realidade. A dimensão estrutural deu-se através da aproximação dos aspectos macroscópicos ou macro-estruturais do processo de capacitação das enfermeiras de UTI. O particular deu-se através da caracterização das enfermeiras de UTI e das práticas de trabalho desenvolvidas nesse espaço. O singular abrangeu os processos Que levam as enfermeiras a buscar sua capacitação, seja ela oferecida pela organização ou fora dela.

Inicialmente, foi captada a realidade objetiva, ou seja, foi feita uma leitura do real, momento em Que foi desvendada a aparência da realidade estudada. Em seguida, a realidade identificada foi interpretada, tentando-se descobrir a essência do processo de capacitação em estudo, com o auxílio das categorias analíticas para o recorte do fenômeno estudado.

\section{RESULTADOS E DISCUSSÃO}

\section{Analisando a Conformação do Processo de Capacitação da Enfermeira de Unidade de Tratamento Intensivo}

Os dados documentais e as falas das enfermeiras entrevistadas evidenciaram um conjunto de significados e significações Que retratam uma dada realidade e convergem para um conjunto de idéias elaboradas a partir das relações estabelecidas pelos sujeitos da investigação, com seu espaço de trabalho, considerando o contexto do fenômeno estudado. Assim, considerando a Questão norteadora deste estudo e os núcleos de sentidos na realização da análise dos conteúdos, conformaram-se as seguintes categorias empíricas: a sofisticação tecnológica impulsionando a capacitação da enfermeira; o individual e o coletivo organizacional na dinâmica da capacitação da enfermeira; a capacitação como instrumento e como produto do processo de trabalho.

\section{A sofisticação Tecnológica Impulsionando a Capacitação da Enfermeira}

A categoria sofisticação tecnológica impulsionando a capacitação da enfermeira, evidenciou o processo de capacitação como um espaço histórico-estrutural, sendo, portanto, um espaço dinâmico, processual, sofrendo as transformações contínuas das Quais as trabalhadoras, nele inseridas, foram as principais articuladoras.

Os dados analisados indicaram Que o SEC da organização estudada, englobado pelas reformulações sociais do setor saúde, buscou, através da oferta de atividades de capacitação das enfermeiras, a garantia da Qualidade da assistência através do desenvolvimento dos membros da equipe e de tecnologias apropriadas para esse fim. Ficou evidenciado Que esse serviço vem desenvolvendo atividades de capacitação, objetivando o atendimento às necessidades do mercado e de viabilidade da transformação organizacional.

Para o desenvolvimento dessas atividades, a organização, atendendo aos paradigmas contemporâneos de gestão empresarial, não apenas percebeu a importância das trabalhadoras no alcance da excelência na produção de bens e serviços e como viabilidade de transformação organizacional, mas, também, apoiou o desenvolvimento do seu Quadro funcional ao relacionar a transformação da capacidade potencial para capacidade real.

O processo de sofisticação tecnológica exigiu, pois, atualização e aquisição de novos conhecimentos, além de habilidades técnicoinstrumentais Que implicaram em mudanças na natureza e no processo de trabalho. Por outro lado, no ambiente hospitalar, a UTI representa um pólo consumidor do substrato da indústria de equipamentos médicos e os trabalhadores desse universo envolvemse num modelo assistencial tecnológico e, conseQüentemente, vêm transformado o seu modelo de organização do trabalho.

Assim, sendo as UTI espaços de trabalho Que exigem ações rápidas para os cuidados específicos e de alta complexidade, fundamentadas no conhecimento científico e interpessoal, as trabalhadoras, também, buscaram melhorar o seu desempenho laboral.

Os depoimentos dos sujeitos desta investigação indicaram Que, ao tempo em Que a organização oferecia atividades de capacitação para suas trabalhadoras, estas, movidas por interesses profissionais e pessoais, também buscaram outras formas de capacitação, tais como: participação em eventos científicos, pesquisa de artigos científicos em Internet, participação em entidades de classe e órgãos públicos, visitas técnicas a outras organizações hospitalares, cursos de pós-graduação, dentre outras.

Estes achados indicaram Que as trabalhadoras, sujeitos desta investigação, considerando a necessidade de Qualificar suas ações, foram movidas a alterar seu comportamento em busca de melhor desempenho, pela necessidade de expor e justificar a própria prática. Essa necessidade não só ativa, mas, também, direciona a enfermeira para o caminho do aperfeiçoamento, melhoria dos serviços prestados e, conseqüentemente, garantia de melhor desempenho no trabalho(7).

Vale destacar Que as falas das entrevistadas apontaram para a realidade da sociedade contemporânea Que tem evidenciado uma prioridade para o desenvolvimento tecnológico, deixando, em plano secundário, aspectos humanos individuais, o Que sugere uma contradição com o processo de capacitação para o cuidado.

As falas evidenciaram, ainda, Que o desenvolvimento do modelo assistencial vem reproduzindo o modelo de produção da sociedade capitalista, onde o aperfeiçoamento é direcionado para o aumento do consumo dos maquinários e da exploração da força de trabalho.

Os aspectos assistenciais referentes à humanização em UTI têm sido evidenciados freqüentemente pela mídia, Que aponta os trabalhadores da saúde, levando-se em conta o senso comum, como sendo pessoas frias, tecnicistas e, muitas vezes, incapazes 
de mostrar afeto pelo paciente e/ou família. Ao se tratar de enfermeiras de UTI, essa realidade torna-se mais evidente, levandose em consideração o ambiente agressivo do espaço de trabalho e o próprio quadro clínico dos pacientes.

Apesar dessas considerações, observou-se Que as enfermeiras investigadas consideraram importante a aquisição de novos conhecimentos na sua capacitação para o cuidado. Ficou evidenciada a intenção de contribuir para o desenvolvimento de novas maneiras de atuar, refletindo sobre o aspecto da humanização no trabalho, particularmente, na UTI, onde "um arsenal de máeuinas e complexidade, prevalece sobre o usuário, Que é relegado a um plano secundário, contrapondo-se ao discurso da assistência humanizada"(8).

Outro aspecto observado foi Que a organização, ao tempo em Que busca priorizar a capacitação de suas trabalhadoras, ela não estimula as atividades de produção e publicação de novos conhecimentos por suas trabalhadoras. O desenvolvimento de pesquisas entre as enfermeiras entrevistadas tem sido incipiente e as poucas atividades de pesquisa existentes não têm sido direcionadas para a publicação de seus achados.

Dentre as enfermeiras da assistência, entrevistadas, todas Que possuem especialização realizaram trabalhos científicos, como reQuisito para a aquisição do título. Entretanto, nenhuma publicou seu trabalho, apesar de reconhecerem Que a prática de Enfermagem requer troca de experiência entre os profissionais.

\section{O Individual e o Coletivo Organizacional na Dinâmica da Capacitação da Enfermeira:}

Os dados levantados apontaram, também, para aspectos individuais e coletivo-organizacionais, influenciando na capacitação da enfermeira.

Ao traçar um paralelo entre os treinamentos oferecidos pela organização em estudo e a freeüência das enfermeiras entrevistadas, percebeu-se uma baixa participação das mesmas. Ficou evidente Que essa baixa participação guarda relação com aspectos organizacionais e individuais.

Dentre os aspectos organizacionais Que influenciaram a capacitação, destacaram-se: ausência de horário destinado para essa finalidade, recursos financeiros escassos, impossibilidade de conciliar outras atividades externas, seja pessoal ou profissional, trabalho de turno, e desmotivação pelo tempo de atuação na área.

Percebeu-se, também, Que o aumento do tempo de atuação na Enfermagem leva a um comprometimento na motivação e no entusiasmo das enfermeiras. A característica de continuidade no trabalho da Enfermagem requer dos membros da equipe o desenvolvimento ininterrupto das ações cuidativas, sendo necessárias escalas de trabalho diurno, noturno, em dias úteis, feriados e finais de semana. Essa característica da assistência, aliada aos fatores estressores próprios da UTI, contribuem negativamente para a busca por programas de capacitação e, também, para os sentimentos de sofrimento no trabalho( ${ }^{(9)}$.

Outra dificuldade foi expressa na conciliação das atividades profissionais com as responsabilidades com os filhos, aspecto vivenciado pela maioria das entrevistadas. É crescente a inserção da força de trabalho feminina no mercado, sobretudo no setor saúde e, mais especificamente, na Enfermagem. Com isso, a mulher tem sofrido sérias restrições pela dupla jornada de trabalho, e a difícil conciliação entre as atividades profissionais e as relativas à maternidade.

Essa duplicidade de papel concorre para o excessivo desgaste físico e mental, contribuindo para Que a enfermeira chegue ao serviço cansada pela fadiga residual e pelas tarefas domésticas realizadas anteriormente $\mathrm{e}^{(10)}$.

Por outro lado, o momento sócio-econômico, a política salarial imposta pelo mercado, aos trabalhadores em geral, também atinge os trabalhadores da saúde. Neste estudo, foi possível evidenciar Que, das enfermeiras Que possuem dois empregos, 90\% possuem filhos. $\mathrm{O}$ fator financeiro, atrelado ao desenvolvimento profissional dessas trabalhadoras, evidencia Que há necessidade de melhoria da infra-estrutura doméstica para Que as mesmas possam exercer sua atividade profissional em condições de considerar, garantir e efetivar seu aperfeiçoamento profissional requerido pelo mercado de trabalho.

Entretanto, os baixos salários da categoria, aliados à responsabilidade de manutenção da família, em sua grande maioria, assumindo a chefia familiar, foram explicitados, por uma parcela das entrevistadas, como elementos que dificultam as possibilidades de desenvolvimento profissional. Ficou evidenciado, Que essas trabalhadoras carecem de um suporte Que lhes possibilitem uma capacitação profissional, perpassando pelo interesse pessoal, o contexto político organizacional e pelos aspectos sócio-econômicos.

Em relação às atividades laborais em horário não comercial, as repercussões são sentidas de diversas formas na vida individual, familiar e social. Na vida social, a ritmicidade tem predomínio no período noturno e nos fins de semana, para as culturais, associativas e de lazer.

O trabalho noturno, desenvolvido pela mulher, está previsto na legislação, entretanto, observa-se Que os valores sociais e de trabalho, como liberdade de movimentação e disponibilidade de tempo, efetivamente, não têm permitido a mesma compatibilização de sua vida pessoal com a profissional. A capacitação profissional é preterida em detrimento aos aspectos organizacionais.

Na organização em Que se processou a investigação foi nítida a formatação de um hospital de ensino. Uma das UTI é campo de estágio, tanto para residência médica como para curso de especialização sob a forma de residência em Enfermagem de Terapia Intensiva. Esse aspecto foi evidenciado, pelas entrevistadas, como fortalecedor e desencadeador de estímulos para a equipe buscar a atualização dos processos técnico-assistenciais e administrativos como instrumentos para melhorar o desenvolvimento das etapas do processo de trabalho.

Notou-se um intercâmbio de experiências através do eixo central, o trabalho, favorecendo a construção dinâmica de novos conhecimentos. Essa realidade imprime, nesses espaços de trabalho, o desenvolvimento de atividades voltadas para a reflexão entre o saber-fazer e o por Que fazer. Essa intercessão dinâmica estabelecida desmistifica o paradigma de Que as enfermeiras de UTI permanecem na superficialidade dos fenômenos, eneuanto as mesmas, muitas vezes, não dão conta de responder ao processo de trabalho Que executam e não refletem sobre o domínio, a habilidade ou o conhecimento alicerçado em teorias científicas.

Esses achados indicaram a necessidade do profissional de enfermagem ter consciência de Que sua formação deve ser complementada para acompanhar o desenvolvimento científico e 
tecnológico, como para dar respostas às demandas sociais e aos desafios organizacionais impostos pela globalização.

Outro aspecto, também considerado, foi a disponibilização de uma carga horária de trabalho anual correspondente a 4\% destinada a treinamento obrigatório instituído pelo Serviço de Enfermagem para as trabalhadoras de Enfermagem. Entretanto, observa-se Que, apesar dessa indicação institucionalizada, ainda não há a estruturação de um programa Que contemple esse percentual de horas durante o ano. Das vinte e cinco enfermeiras de assistência Que foram Questionadas acerca do conhecimento dessa carga horária, apenas 20\% reconheceram essa disponibilização. Isso denota uma falta de conhecimento dos documentos Que norteiam o funcionamento organizacional e a política de desenvolvimento das trabalhadoras, ao mesmo tempo em Que retrata pouca divulgação dessa normatização entre as enfermeiras.

Além disso, a organização estudada destina, para categorias de nível superior, 30 horas ao ano para participação em eventos externos, fora do ambiente organizacional. Frente a essa Questão, foi evidenciada uma falta de coerência entre a disponibilidade da carga horária e a falta de condições para Que as trabalhadoras possam desfrutar desse direito.

Constatou-se um descompasso entre a especificidade e a necessidade, com o surgimento, no interior das UTI, de movimentos de organização de processos educativos desvinculados de um projeto coletivo e sem condições concretas de contribuir com outros setores Que apresentem as mesmas necessidades.

Evidenciou-se, também, Que a participação das trabalhadoras, no programas de capacitação, se deu a partir da identificação das necessidades de aprendizagem Que elas desenvolviam nas UTI, além da vinculação da viabilidade, na escala de trabalho, de trocas de plantões.

Essa realidade indicou Que os critérios de necessidade de aprendizagem não estão articulados aos aspectos organizativos e gerenciais.

Outro aspecto evidenciado, além da necessidade de aprendizagem, foi o interesse pessoal, considerado como um dos principais motivos para a participação em programas de aperfeiçoamento. Nesta direcionalidade, entende-se que a motivação humana no trabalho precisa ser compreendida no contexto real em Que a trabalhadora está inserida. A falta de interesse pode ser um sinalizador significativo para a organização rever seus processos gerenciais e sua política de pessoal.

Outro fato identificado foi Que a estruturação do processo de capacitação, na organização estudada, não se efetua a partir de um planejamento estratégico da organização.

\section{A Capacitação como Instrumento e como Produto do Processo de Trabalho}

Através dessa categoria, ficou explicitado Que a capacitação se constitui em elemento primordial das práticas e dos processos de mudança e, conseqüentemente, impulsionadora para a transformação das enfermeiras como sujeitos sociais, ou seja, como pessoas Que buscam autonomia, Que estão dispostas a correr riscos, abrir-se ao novo na perspectiva de ser alguém capaz de perceber seu papel profissional/social, frente aos desafios colocados a cada momento e engajando-se com o Que se passa ao seu redor. Nesse processo de transformação, efetiva-se a interligação de elementos como a participação, o conflito e a cooperação ${ }^{(11)}$.

No presente estudo, ficou evidenciado Que as enfermeiras de assistência não conseguem participar dos treinamentos internos no seu horário de trabalho, enquanto Que as enfermeiras coordenadoras, teoricamente, conseguem estabelecer a distribuição das suas atividades, de forma a contemplar sua capacitação no espaço intramural.

Quando se compreende Que o trabalho coletivo é estimulado a partir de metas comuns, não se concebe tais comportamentos privilegiadores, uma vez que é através do assistir Que a enfermeira redireciona os processos de cuidar, influenciando nos resultados do processo de trabalho, por ser ela, a trabalhadora, capaz de desempenhar e avaliar esse processo( ${ }^{(9)}$.

A análise dos dados possibilitou, também, a identificação do elemento de cooperação como possibilitador da transformação do cuidado. E essa transformação da prática das enfermeiras foi apontada como relacionada ao processo de capacitação Que possibilita a transformação do produto do trabalho prestado à sociedade.

O esforço das trabalhadoras desenha e, ao mesmo tempo, transforma o produto do trabalho prestado à sociedade, ou seja, o fortalecimento das trabalhadoras como sujeitos sociais capazes de conduzir projetos coletivos de mudança ${ }^{(12)}$. Essa condução é dada, enfrentando conflitos, negociando e disputando colocações nas estruturas sociais, através do desempenho no processo de trabalho.

Através da sua capacitação, a enfermeira atua sobre o objeto do seu trabalho, ou seja, sobre aquilo que se trabalha e que contem em si a potencialidade do produto ou serviço em Que irá ser transformado pela ação da trabalhadora. A enfermeira ao se capacitar, ela é transformada por esse aprendizado e conquista condições plenas de agir sobre o seu objeto, sendo agente de transformação do seu trabalho ${ }^{(13)}$.

Os dados analisados revelaram, ainda, Que a geração de conhecimentos torna o indivíduo bem informado, entretanto, o valor desse conhecimento vincula-se à administração, mobilização e articulação em favor da resolução dos desafios profissionais. Para isso, são exigidas novas habilidades de raciocínio, como criatividade, capacidade de tomar decisões, saber pensar e aprender a aprender. Ou seja fazer a articulação de uma informação com outra para gerar um novo conhecimento.

Essa dinâmica demonstra a articulação entre os elementos Que se conjugam e formam as relações internas e externas, sob a forma de cuidado, com as enfermeiras e com os usuários sob a forma de cuidado, respectivamente.

\section{CONSIDERAÇÕES FINAIS}

Nessa investigação foi possível conhecer as convergências e as divergências Que caracterizam o processo de capacitação de enfermeiras numa realidade concreta.

As enfermeiras de UTI, sujeitos do presente estudo, têm, na conformação dos seus processos de capacitação profissional, determinações históricas e sociais Que se interpenetram com as pessoais. Isto ocorre porque delas são requeridos saberes Que favoreçam um desempenho eficaz, frente à diversidade de situações complexas e mutáveis. Por outro lado, as ações por elas desempenhadas devem garantir baixo custo, aumento de 
produtividade e manutenção da Qualidade.

A capacitação dessas enfermeiras vem sendo influenciada pelas transformações Que a sociedade passa, nos aspectos sócio-político e econômico, e, por conseguinte, pelas mudanças na natureza e no processo de trabalho.

A organização hospitalar, estudada, favorece o desenvolvimento do processo de capacitação das enfermeiras. Entretanto, a mesma organização possui contradições na estruturação das políticas de desenvolvimento, Que, efetivamente, dificulta a viabilidade do processo de capacitação dessas enfermeiras, tanto no espaço intramural, como no extramural.

Essas contradições estão relacionadas aos aspectos políticos, administrativos e gerenciais Que permeiam esse processo. Este por sua vez, relaciona-se com as reformulações sociais e empresariais.

Os programas internos de capacitação para as enfermeiras de UTI têm acontecido, em sua grande maioria, de forma pontual, momentâneos e descontextualizados das especificidades da área estudada, ou seja da UTI.

O processo de capacitação profissional Que a organização desenvolve privilegia os aspectos técnicos em detrimentos dos administrativos e psicossociais Que permeiam o trabalho em UTI, configurando um corpo de saberes Que valoriza a construção de conhecimentos estáticos, pouco reflexivos e voltados, eminentemente, para a padronização do processo de trabalho e/ ou consolidação de protocolos de Enfermagem.

Apesar das três UTIs, campos dessa investigação, fazerem parte do mesmo complexo hospitalar, foi possível descrever algumas particularidades entre elas e perceber a desarticulação das atividades educacionais Que cada uma desenvolve, não sendo viabilizada a troca de experiências entre estas unidades.

A enfermeira de UTI reconhece Que sua capacitação profissional Ihe instrumentaliza para os desafios impostos pelas mudanças de cenários sociais, políticos e econômicos com uma influência, mais imediata, da evolução tecnológica e epidemiológica.

Compreende-se Que o suporte organizacional representa um fator preponderante no desenvolvimento do processo de capacitação da enfermeira. Entretanto, é necessário que a trabalhadora reconheça essa necessidade e busque investir em sua formação intelectual, cognitiva e interpessoal, características de relevância no desempenho de suas funções na UTI.

Quanto ao produto do processo de capacitação, ficou evidenciado Que, Quando a capacitação é desenvolvida pelas enfermeiras, contribui favoravelmente para um melhor desempenho laboral das trabalhadoras, refletindo-se na melhoria dos serviços prestados.

Essa mudança comportamental também foi apontada como uma resposta à exigência mercadológica. Ficou constatado Que a enfermeira de UTI vem assumindo uma posição diante dos desafios encontrados na sua prática social, cujas tendências exigem do profissional um perfil capaz de investir no seu autoconhecimento.

Por se tratar de um hospital com perfil nosológico que desperta interesse científico, o ambiente favorece o desenvolvimento de pesquisa entre essas trabalhadoras.

$\mathrm{Na}$ medida em Que a enfermeira for compreendida na sua totalidade, e não apenas como força de trabalho, estará maximizando seu processo de capacitação e, conseQüentemente, tornando-se mais comprometida com o produto do seu trabalho em benefício dos interesses organizacionais.

Pelo exposto, percebeu-se Que o processo de capacitação profissional das enfermeiras de UTI representa, pois, um conjunto de práticas e saberes intrinsecamente relacionados à totalidade social, nos contextos individuais de Quem promove e de Quem procura essas práticas e saberes.

O processo de capacitação profissional da enfermeira não se constitui, pois, numa esfera marginal e autônoma. Ele se articula de acordo com a forma como o setor saúde está articulado ao mundo da produção e do trabalho.

O modelo de gestão focado na educação, potencializa o aprendizado das pessoas, contribui para o desenvolvimento de conhecimentos diversificados, voltados para os resultados das organizações.

No contexto de globalização, as organizações são colocadas, a todo o momento, frente a novas informações, novas oportunidades e novos desafios para Que elevem seus potenciais e resultados.

A competitividade do mercado e o foco no conhecimento despertam, nos trabalhadores, os interesses pelo aperfeiçoamento, atendendo à necessidade da organização, da sociedade e deles próprios como profissionais.

Além do conhecimento da satisfação Que a trabalhadora tem do trabalho, a estruturação dos programas de capacitação deve inspirar-se nos pressupostos de Que o homem é sujeito, portanto, produto de um meio social.

\section{REFERÊNCIAS}

I. Deluiz N. Qualificação, competências e certificação: visão do mundo do trabalho. Formação 200 I; I (2): 3-15.

2. Deluiz N. Formação do trabalhador: produtividade e cidadania. Rio de Janeiro: Shape; 1995.

3. Costa MT. Comprometimento da enfermeira com a organização hospitalar e com a carreira: um estudo de caso em uma organização hospitalar [dissertação]. Salvador (BA): Escola de Enfermagem, Universidade Federal da Bahia; 1999.

4. Saupe R, organizadora. Educação em enfermagem: da realidade construída à possibilidade em construção. Florianópolis (SC): UFSC; 1998.

5. Triviños ANS. Introdução à pesQuisa em ciências sociais: a pesquisa Qualitativa em educação. São Paulo: Atlas: 1992.
6. Bardin L. Análise de Conteúdo. Lisboa (PO): Edições 70, 1979.

7. Pereira LL. Participação do enfermeiro em programas de aperfeiçoamento [dissertação]. São Paulo (SP): Escola de Enfermagem, Universidade de São Paulo; 1992.

8. Hayashi AAM, Gisi ML. O cuidado de enfermagem no CTI: da ação-reflexão à conscientização. Texto Contexto Enferm 2000; 9(2): 824-37.

9. Fernandes ID, Araújo FA, Fernandes I, Reis LS, Gusmão MCCM, Santana N. Competência interpessoal nas práticas em saúde: o individual e o coletivo organizacional. Texto Contexto Enferm 2003; 12(2): 210-5.

10. Corrêa AK. Sendo enfermeira no centro de terapia intensiva. Rev Bras Enferm 1995; 48(3): 233-41. 
11. L'Abbate S. Agente de trabalho/sujeitos? Repensando a capacitação de recursos humanos em saúde coletiva. In: Canesqui SM (org). Dilemas e dasafios das Ciências Sociais na Saúde Coletiva. São Paulo: HUCITEC; 1995. p. 15 I-6I
12. Melo MLC, Nascimento MAA. Treinamento introdutório para enfermeiras dirigentes: possibilidades para gestão do SUS. Rev Bras Enferm 2003; 56(6): 674-7.

13. Sanna MC. Os processos de trabalho em enfermagem. Rev Bras Enferm 2007; 60(20): 22 I-4. 